\title{
Resynchronization Therapy in the Elderly
}

\author{
Luca Bontempi, Francesca Vassanelli and Antonio Curnis
}

Unit of Cardiology, University of Brescia, Spedali Civili Hospital, Brescia, Italy

DOI: http://doi.org/10.17925/EJAE.2016.02.01.18

\begin{abstract}
Implantable cardioverter-defibrillators (ICDS) are increasingly used in patients $\geq 80$ years old, and the proportion of devices incorporating cardiac resynchronization therapy (CRT) increases with patient age. In this era of patient-centered care, important questions arise about the patterns of use, clinical outcomes and cost-effectiveness of CRT for older patients. Currently, few data are available that specifically address the effects of CRT in the elderly.
\end{abstract}

\section{Keywords}

Resynchronization therapy, heart failure, elderly patients

\begin{abstract}
Disclosure: Luca Bontempi, Francesca Vassanelli and Antonio Curnis have nothing to disclose in relation to this article. No funding was received in the publication of this article. This article is a short opinion piece and has not been submitted to external peer reviewers

Open Access: This article is published under the Creative Commons Attribution Noncommercial License, which permits any noncommercial use, distribution, adaptation, and reproduction provided the original author(s) and source are given appropriate credit.

Received: 3 March 2016 Published Online: 10 May 2016 Citation: European Journal of Arrhythmia \& Electrophysiology, 2016;2(1):18-9

Correspondence: Luca Bontempi, Piazzale Spedali Civili 1, 25100, Brescia, Italy; E: bontempiluca@libero.it
\end{abstract}

The prevalence of congestive heart failure (HF) increases with age: $\mathrm{HF}$ is implicated in $20 \%$ of hospital admissions in those aged $>65$, and octogenarians are 20 times more likely to be hospitalised for HF than younger patients. ${ }^{1}$ As a consequence, elderly patients currently represent a larger portion of patients with HF, but they substantially differ from younger patients in both concurrent comorbid conditions and prescribed medical therapy. Cardiac resynchronization therapy (CRT) has been shown to improve symptoms and cardiac function, decrease cardiovascular and arrhythmic mortality and HF events in patients with wide QRS, low left ventricular ejection fraction (LVEF), and is also effective in less symptomatic patients. Therefore, CRT is a well-established therapy for HF patients, and its indications have recently been extended to patients in New York Heart Association (NYHA) class II. ${ }^{2}$

Implantable cardioverter-defibrillators (ICDS) are increasingly used in patients $\geq 80$ years old, and the proportion of devices incorporating CRT increases with patient age, reaching up to $40 \%$ in octogenarians. ${ }^{3-5}$ In this era of patient-centered care, important questions arise about patterns of use, clinical outcomes and cost-effectiveness of CRT for older cardiac patients. In particular, the use of CRT in patients with multiple illnesses, frailty and cognitive impairment is not known.

Currently, few data are available that specifically address the effects of CRT in the elderly. Octogenarians and nonagenarians have not been systematically excluded from multicentre, randomised clinical trials evaluating CRT, and guidelines discourage the use of age alone as an exclusion for implantation. However their numbers in these studies have been small. Furthermore, many clinical trials have excluded patients with multiple comorbidities, which are more likely to occur in geriatric patients. In studies of CRT for severe HF, the median age of patients in major studies was 64-67 years.
Sub-group analysis of the Comparison of Medical Therapy, Pacing and Defibrillation in Heart Failure (COMPANION) and the Cardiac Resynchronization - Heart Failure (CARE-HF) trials showed a benefit from CRT with an ICD (CRT-D) relative to CRT with a pacemaker (CRT-P) and a benefit of CRT-P compared with optimised medical therapy, respectively, in subjects $>65$ years old in terms of death or hospitalisations. ${ }^{6-8}$

Subgroup age specific analyses from more recent trials of the use of CRT in patients with less severe HF show contrasting results. The Resynchronization-Defibrillation for Ambulatory Heart Failure Trial (RAFT) ${ }^{9}$ and Resynchronization Reverses Remodeling in Systolic Left Ventricular Dysfunction (REVERSE) ${ }^{10}$ trial reported non-significant trends towards benefit of primary endpoints of death or hospitalisation for CRT-D versus ICD alone among subjects $>65$ years and no difference in HF clinical composite response between CRT-on and CRT-off in elderly patients, respectively. In the Multicenter Automatic Defibrillator Implantation Trial - Cardiac Resynchronization Therapy (MADIT-CRT) subgroup analysis it was found that CRT-D significantly reduced in the composite endpoint (death or HF) among subjects aged 60-74 years and $\geq 75$ years, and this reduction was not significant in patients younger than 60 years. ${ }^{11}$ It must be emphasised, however, that the majority of those studies were not a priori designed to examine survival or HF hospitalisation in older patients nor were they statistically powered to do so.

Moreover, it is important to highlight that the benefits of use of CRT in older patients extends beyond the typical hard primary endpoints (death, cardiac events, hospitalisations) and include other outcomes that may be equal or greater concern to this population, such as quality of life (QoL), cognitive and functional status, and end-of-life experiences. In elderly patients enrolled in the Multicenter InSync Randomized Clinical Evaluation (MIRACLE) and MIRACLE-ICD trials (174 patients above the age of 75 years) CRT resulted in significant 
improvements in NYHA functional class and LVEF, regardless of age, but improvement in other clinical (e.g., QoL) or echocardiographical end points was not found to be significant. ${ }^{12,13}$ Elderly patients with HF often have multiple comorbidities that affect QoL, beyond the reach of CRT therapy. However, functional and symptom limitations in HF worsen overall QoL and well-being via reducing the ability to participate fully in the rehabilitative process, the activities of daily living and recreation, and social relationships across conditions.

In addition, procedural complications are higher in that implantation of CRT devices than for ICDS, particularly in older versus younger patients ( $<65$ years $2.8 \%$ and $>85$ years $4.5 \%$, overall response [OR] 1.15). ${ }^{14}$ Complications not only impact individual patients, but also increase the financial cost of device implantation. Future research should focus on the effectiveness of CRT-D versus that of CRT-P in reducing HF symptoms, improving myocardial function, reducing hospitalisation, and/or improving survival in elderly patients candidates for CRT. There is also a need to identify clinical predictors of response in patients who are deemed appropriate candidates for CRT-P or CRT-D devices. ${ }^{15}$

To date, the decision to implant a CRT-D or a CRT-P does not take into account the age of the patient, but rather 1-year life expectancy. ${ }^{2}$ Physicians should fully discuss the implications of CRT with older patients including the benefits of implantation and the possibility of complications (eg. phrenic nerve stimulation) or inappropriate shocks. such discussions should take into account the patient's health care goals and priorities, including quality versus quantity of life, avoidance of invasive procedures and tolerance of uncertainty.
1. Jessup $\mathrm{M}$, Brozena $\mathrm{S}$, Heart failure, N Eng/ J Med, 2003;348:2007-18.

2. Brignole M, Auricchio A, Baron-Esquivias G, et al., 2013 ESC Guidelines on cardiac pacing and cardiac resynchronization therapy, Eur Heart J, 2013;34:2281-329.

3. Kramer DB, Reynolds MR, Mitchell SL, Resynchronization: considering device based cardiac therapy in older adults, I Am Geriatr Soc, 2013:61:615-21.

4. Barra S, Providencia R, Paiva L, et al., Implantable cardioverterdefibrillators in the elderly: rationale and specific age-related considerations, Europace, 2015:17:174-86.

5. Chen J, Hocini M, Larsen TB, et al., Scientific Initiative Committee, European Heart Rhythm Association. Clinical management of arrhythmias in elderly patients: results of the European Heart Rhythm Association survey, Europace, 2015;17:314-7.

6. Abraham WT, Fisher WG, Smith AL, et al., Cardiac resynchronization in chronic heart failure, $N$ Engl I Med 2002;346:1845-53

7. Bristow MR, Saxon LA, Boehmer J, et al., Cardiac- resynchronization therapy with or without an implantable defibrillator in advanced chronic heart failure, $N$ Engl I Med 2004;350:2140-50.

8. Cleland JG, Daubert JC, Erdmann E, et al., The effect of cardiac resynchronization on morbidity and mortality in heart failure, N Eng/ J Med, 2005;352:1539-49.

9. Tang AS, Wells GA, Talajic M, et al., Cardiac-resynchronization therapy for mild-to-moderate heart failure, N Eng/ I Med, 2010: 363.2385-95.

10. Daubert C, Gold MR, Abraham WT, et al., Prevention of disease progression by cardiac resynchronization therapy in patients with asymptomatic or mildly symptomatic left ventricular dysfunction: insights from the European cohort of the REVERSE (Resynchronization Reverses Remodeling in Systolic Left Ventricular Dysfunction) trial, J Am Coll Cardiol, 2009;54:1837-46.

11. Penn J, Goldenberg I, Moss AJ, et al., Improved outcome with preventive cardiac resynchronization therapy in the elderly: A MADIT-CRT substudy, J Cardiovasc Electrophysiol, $2011 ; 22: 892-7$
12. Kron J, Aranda JM Jr., Miles WM, et al., Benefit of cardiac resynchronization in elderly patients: Results from the multicenter Insync randomized clinical evaluation (MIRACLE) and multicenter Insync ICD randomized clinical evaluation (MIRACLE-ICD) trials, J Interv Card Electrophysio , 2009:25:91-6.

13. Kron J, Conti JB, Cardiac resynchronization therapy for treatment of heart failure in the elderly, Heart Fail Clin, 2007:3:511-8.

14. Tsai $\mathrm{V}$, Goldstein MK, Hsia HH, et al., Influence of Age on Perioperative Complications Among Patients Undergoing Implantable Cardioverter-Defibrillators for Primary Prevention in the United States, Circ Cardiovasc Qual Outcomes, 2011;4:549-56.

15. Agency for Healthcare Research and Quality. Evidence-based practice center systematic review protocol: use of cardiac resynchronization therapy in the Medicare population. U.S. Department of Health and Human Services/ARHQ Available at: http://www.ahrq.gov/research/findings/ta/ topicrefinement/crt protocol.pdf (accessed 31 March 2016). 